\title{
Alina Marchlewska
}

\section{ON A GENERALIZATION OF CLOSE-TO-CONVEXITY FOR COMPLEX HOLOMORPHIC FUNCTIONS IN $\mathrm{C}^{n}$}

\footnotetext{
Abstract. Sveral authors (I. I. Bawrin [1], K. Dobrowolska, I. Dziubiński, P. Liczberski, R. Sitarski [3], [4], [5], [13], S. Gong, S. S. Miller [6], Z. J. Jakubowski and J. Kamiński [8], J. Janiec [10] and others) studied various families of complex holomorphic functions in $\mathbf{C}^{n}$ and in Banach space, corresponding with famous subclasses of univalent functions. In this paper we study a class of holomorphic functions of $n$ complex variables analogous to the class of close-to-convex functions of one variable considered by M. Biernacki, W. Kaplan and Z. Lewandowski (see [2], [11], [12]).
}

\section{Introduction}

Let $\Omega$ be a bouded complete circular and convex domain in $\mathbf{C}^{n}$. If we put

$$
\|\omega\|=\inf \left\{c>0: \frac{1}{c} \omega \in \Omega\right\}, \quad \omega \in \mathbf{C}^{n},
$$

then $\left(\mathbf{C}^{n},\|\cdot\|\right)$ is a Banach space, $\Omega$ is a unit ball in this space and $\partial \Omega$ is a unit sphere (see e.g. [14]). In the paper we use this norm.

Let $I: \mathbf{C}^{n} \rightarrow \mathbf{C}$ be the following linear functional

$$
I(z)=z_{1}+z_{2}+\ldots+z_{n}, \quad z=\left(z_{1}, z_{2}, \ldots z_{n}\right) \in \mathbf{C}^{n}
$$

and let

$$
\chi(I)=\left\{z \in \mathbf{C}^{n}: I(z) \neq 0\right\}, \quad \gamma(I)=\mathbf{C}^{n} \backslash \chi(I) .
$$

Clearly, $\gamma(I)$ is a hyperplane, $\chi(I)$ is dense in $\mathbf{C}^{n}$ and $\chi(I) \cap \partial \Omega$ is dense in $\partial \Omega$.

Let $H(\Omega)$ denote the class of all holomorphic functions $f: \Omega \rightarrow \mathbf{C}$ of the form

$$
f(z)=I(z)+\sum_{m=2}^{\infty} P_{m, f}(z), \quad z \in \Omega,
$$

1991 Mathematics Subject Classification: Primary 32A10, 30C45.

Key words and phrases: circular domains, holomorphic functions, close-to-convexity. 
where $P_{m, f}(z)=\frac{1}{m !} D^{m} f(0)(z, \ldots, z)$, and $D^{m} f(z)$ is the $n$-th Frechet derivative of the function $f$ at the point $z$, while $D^{m} f(0)\left(w^{1}, \ldots, w^{m}\right)$ is the value of $D^{m} f(z)$ on the vectors $w^{1}, \ldots, w^{m} \in \mathbf{C}^{n}$.

There were examined (see [10]) the following families of functions

$$
\begin{aligned}
& S^{*}(\Omega)=\{f \in H(\Omega):\left.f(z) \neq 0, \operatorname{Re} \frac{D f(z)(z)}{f(z)}>0, z \in \chi(I) \cap \Omega\right\}, \\
& S^{c}(\Omega)=\{f \in H(\Omega): D f(z)(z) \neq 0, \\
&\left.\operatorname{Re}\left(1+\frac{D^{2} f(z)(z, z)}{D f(z)(z)}\right)>0, z \in \chi(I) \cap \Omega\right\} .
\end{aligned}
$$

In the geometric theory of functions on the complex plane well known is the class $S$ of functions $F(\zeta)=\zeta+a_{2} \zeta^{2}+\ldots$, holomorphic and univalent in the unit disc $\Delta=\{\zeta \in \mathbf{C}:|\zeta|<1\}$ and its subclasses $S^{*}$ (starlike functions), $S^{c}$ (convex functions) and $S^{c c}$ (close-to-convex functions).

The definitions of the families $S^{*}(\Omega)$ and $S^{c}(\Omega)$ are based on the analytical conditions: $\operatorname{Re} \frac{\zeta F^{\prime}(\zeta)}{F(\zeta)}>0$ for $F \in S^{*}$ and $\operatorname{Re}\left(1+\frac{\zeta F^{\prime \prime}(\zeta)}{F^{\prime}(\zeta)}\right)>0$ for $F \in S^{c}$ (see [10]).

In this paper we shall consider the family $S^{c c}(\Omega)$ which is connected with the class $S^{c c}$ in a similar way. We say that $f \in H(\Omega)$ belongs to the class $S^{c c}(\Omega)$ if there exists a function $g \in S^{*}(\Omega)$ such that

$$
\operatorname{Re} \frac{D f(z)(z)}{g(z)}>0, \quad z \in \chi(I) \cap \Omega .
$$

We say that functions $f \in S^{c c}(\Omega)$ and $g \in S^{*}(\Omega)$ are associated.

Let $h \in H(\Omega)$ and $g(z)=D h(z)(z)$ for $z \in \Omega$. By generalization of Alexander's Theorem (see [10]), we know that $h \in S^{c}(\Omega)$ if and only if the function $g$ belongs to $S^{*}(\Omega)$, so from (2), $f$ belongs to $S^{c c}(\Omega)$ if and only if, there exists a function $h \in S^{c}(\Omega)$ such that

$$
\operatorname{Re} \frac{D f(z)(z)}{D h(z)(z)}>0, \quad z \in \chi(I) \cap \Omega \text {. }
$$

Analogously as below, we say that functions $f \in S^{c c}(\Omega)$ and $h \in S^{c}(\Omega)$ are associated.

Now we prove the announced relation between tha classes $S^{c c}(\Omega)$ and $S^{c c}$. Proposition 1. If $f \in S^{c c}(\Omega)$, then for any $w \in \chi(I) \cap \partial \Omega$ the function $F_{w}$,

$$
F_{w}(\zeta)=\frac{f(\zeta w)}{I(w)}, \quad \zeta \in \Delta,
$$

is close-to-convex $\left(F_{w} \in S^{c c}\right)$.

Proof. Let us fix $f \in S^{c c}(\Omega)$ and let $g \in S^{*}(\Omega)$ be a function associated with $f$. Then the function $G_{w}(\zeta)=\frac{g(\zeta w)}{I(w)}$ belongs to $S^{*}$ (see [10]) and 
from (2) we obtain

$$
0<\operatorname{Re} \frac{D f(\zeta w)(\zeta w)}{g(\zeta w)}=\operatorname{Re} \frac{\zeta[D f(\zeta w)(w)]}{g(\zeta w)}=\operatorname{Re} \frac{\frac{\zeta D f(\zeta w)(w)}{I(w)}}{\frac{g(\zeta w)}{I(w)}}
$$

for $w \in \chi(I) \cap \partial \Omega$ and $\zeta \in \Delta$. In view of (4) we have for $w \in \chi(I) \cap \partial \Omega$

$$
F_{w}^{\prime}(\zeta)=\frac{D f(\zeta w)(w)}{I(w)}, \quad \zeta \in \Delta
$$

thus

$$
\operatorname{Re} \frac{\zeta F_{w}^{\prime}(\zeta)}{G_{w}(\zeta)}>0, \quad \zeta \in \Delta,
$$

and we conclude that $F_{w} \in S^{c c}$ (see [12], [11]).

Proposition 2. $S^{c c}(\Omega)$ is a proper superclass of $S^{*}(\Omega)$.

Proof. The inclusion $S^{*}(\Omega) \subset S^{c c}(\Omega)$ follows from the definitions of $S^{*}(\Omega)$ and $S^{c c}(\Omega)$. Indeed, if $f \in S^{*}(\Omega)$, then for $z \in \chi(I) \cap \partial \Omega$ we have that (2) is fulfilled with $g=f$. To prove that $S^{*}(\Omega) \neq S^{c c}(\Omega)$ we consider the function $f_{\alpha}(z): \Omega \rightarrow \mathbf{C}$ of the form

$$
f_{\alpha}(z)=\frac{I(z)\left(1-A Z_{w}^{*}(z)\right)}{\left(1-B Z_{w}^{*}(z)\right)^{2}}
$$

where $Z_{w}^{*}(z)=\frac{I(z)}{I(w)}, A=e^{2 i \alpha} \cos \alpha, B=e^{i \alpha}, \alpha \in(0, \pi)$ and $w \in \chi(I) \cap \partial \Omega$ is such that $|I(w)|=\max _{z \in \bar{\Omega}}|I(z)|$. Let us notice that $Z_{w}^{*}$ is a functional over $\mathbf{C}^{n}\left(Z_{w}^{*} \in \mathbf{C}^{n *}\right)$ such that

$$
Z_{w}^{*}(w)=1 \text { and }\left\|Z_{w}^{*}\right\|=1
$$

We will prove that $f_{\alpha} \in S^{c c}(\Omega)$ but for $\alpha \neq \pi / 2, f_{\alpha} \notin S^{*}(\Omega)$. Let $F_{w}$ be defined by (4) for $f=f_{\alpha}$. To show that $f_{\alpha} \notin S^{*}(\Omega)$ it suffices to prove that $F_{w} \notin S^{*}$ (see [10]).

From the definitions of $f_{\alpha}$ and $F_{w}$ we have

$$
F_{w}(\zeta)=\frac{\zeta\left(1-A Z_{w}^{*}(\zeta w)\right)}{\left(1-B Z_{w}^{*}(\zeta w)\right)^{2}}=\frac{\zeta(1-A \zeta)}{(1-B \zeta)^{2}}, \quad \zeta \in \Delta
$$

Thus $F_{w}$ maps $\Delta$ onto the complex plane minus a vertical slit that starts at

$$
s_{0}=\frac{-i e^{2 i \alpha}}{4 \sin \alpha}
$$

and extends upward to $-(\cos \alpha) / 2+i \infty$ (see [6], vol I, p. 196). For $\alpha \neq$ $\pi / 2$, we have $\operatorname{Re} s_{0} \neq 0$, and $F_{w}(\Delta)$ is not starlike. Then $F_{w} \notin S^{*}$ and consequently $f_{\alpha} \notin S^{*}(\Omega)$ (see $[10]$ ). 
To show that $f_{\alpha} \in S^{c c}(\Omega)$ let us consider the associated function $g_{\alpha} \in S^{*}(\Omega)$ of the form

$$
g_{\alpha}(z)=\frac{I(z)}{\left(1-B Z_{w}^{*}(z)\right)^{2}}, \quad z \in \Omega .
$$

For $\alpha \neq \pi / 2$ we have

$$
\operatorname{Re} \frac{D f_{\alpha}(z)(z)}{g_{\alpha}(z)}>0, \quad z \in \chi(I) \cap \Omega .
$$

Indeed, from definition of $f_{\alpha}$ we obtain

$$
D f_{\alpha}(z)(z)=\frac{1+B \frac{I(z)}{I(w)}-2 A \frac{I(z)}{I(w)}}{\left(1-B \frac{I(z)}{I(w)}\right)^{3}} I(z)
$$

so for $\alpha \neq \pi / 2$

$$
\frac{D f_{\alpha}(z)(z)}{g_{\alpha}(z)}=1-\frac{2}{i \frac{I(w)}{I(z)}+1}=1-\frac{2\left(1+i \frac{\overline{I(w)}}{I(z)}\right)}{\left|1+i \frac{I(w)}{I(z)}\right|^{2}} .
$$

Thus (6) is true if and only if

equivalently

$$
1-\frac{2\left(1-\operatorname{Im} \frac{I(w)}{I(z)}\right)}{\left|1+i \frac{I(w)}{I(z)}\right|^{2}}>0, \quad z \in \chi(I) \cap \Omega
$$

$$
\left|\frac{I(w)}{I(z)}\right|^{2}>1, \quad z \in \chi(I) \cap \Omega .
$$

The last inequality is obvious by the Maximum Principle and by the choice of the point $w$. In consequence we obtain that $f \in S^{c c}(\Omega)$.

THEOREM 1. If $f \in S^{c c}(\Omega)$ and $w \in \partial \Omega$, then for $m \in \mathbf{N}$

$$
\left|P_{m, f}(w)\right| \leq m|I(w)| \text {. }
$$

The above bound is sharp. The equality is attained by the function $\tilde{f} \in S^{c c}(\Omega)$ of the form

$$
\tilde{f}(z)=\frac{I(z)}{\left(1-Z_{w}^{*}(z)\right)^{2}},
$$

where $Z_{w}^{*} \in\left(\mathbf{C}^{n}\right)^{*}$ satisfies the conditions $(5)$.

Proof. Let first $w \in \chi(I) \cap \partial \Omega$. Then from (1) for function $F_{w}$ defined by (4) we have

$$
F_{w}(\zeta)=\frac{I(\zeta w)+\sum_{m=2}^{\infty} P_{m, f}(\zeta w)}{I(w)}=\zeta+\sum_{m=2}^{\infty} \frac{P_{m, f}(w)}{I(w)} \zeta^{m}, \quad \zeta \in \Delta .
$$


From Proposition 1 it follows that $F_{w} \in S^{c c}$, so from the coefficient estimates in the class $S^{c c}$ (see [6]) we have

$$
\left|\frac{P_{m, f}(w)}{I(w)}\right| \leq m, \quad m \in \mathbf{N} \backslash\{1\} .
$$

Hence, we have (7) for $w \in \chi(I) \cap \partial \Omega$.

Let us assume now that $w \in \gamma(I) \cap \partial \Omega$. In view of density $\chi(I) \cap \partial \Omega$ in $\partial \Omega$, there exists $w=\lim _{k \rightarrow \infty} w^{k}$, where $w^{k} \in \chi(I) \cap \partial \Omega, k \in \mathbf{N}$. Accordingly to the first case we get

$$
\left|P_{m, f}\left(w^{k}\right)\right| \leq m\left|I\left(w^{k}\right)\right|
$$

Therefore, by the continuity of $P_{m, f}, I$ and $|\cdot|$, letting $k \rightarrow \infty$ we obtain (7).

Now, we will prove the sharpness of (7). Let us observe, that the function $\tilde{f}$ defined by (8) has the following expansion

$$
\tilde{f}(z)=I(z)+\sum_{m=2}^{\infty} m I(z)\left(Z_{w}^{*}(z)\right)^{m-1}, \quad z \in \Omega .
$$

By the fact that $\tilde{f} \in S^{c c}(\Omega)$ and by (1), in view of the identity theorem for series of homogeneous polynomials, we have

$$
P_{m, \tilde{f}}(z)=m I(z)\left(Z_{w}^{*}(z)\right)^{m-1} \text { for } m \in \mathbf{N} \backslash\{1\} .
$$

Since $r w \in \Omega$ for $r \in(0,1)$, then putting $z=r w$ we get

$$
P_{m, \tilde{f}}(r w)=m I(r w)\left(Z_{w}^{*}(r w)\right)^{m-1} \text { for } m \in \mathbf{N} \backslash\{1\} .
$$

Then, in view of m-homogeneity $P_{m, \bar{f}}$ and (5), we have

$$
P_{m, \tilde{f}}(w)=m I(w), \quad m \in \mathbf{N} \backslash\{1\}
$$

for $w \in \gamma(I) \cap \partial \Omega$.

From the above theorem we obtain

Corollary 1. If $f \in S^{c c}(\Omega)$, then $f(z)=0$ for $z \in \gamma(I) \cap \Omega$.

Corollary 2. If $f \in S^{c c}(\Omega)$, then $\left\|P_{m, f}\right\| \leq m\|I\|$, where $\left\|P_{m, f}\right\|=$ $\max _{\|x\|=1}\left|P_{m, f}(x)\right|$. This estimate is sharp and attained by the function defined by (8).

Theorem 2. Let $f \in S^{c c}(\Omega)$ and $z \in \Omega$. Then

$$
\frac{|I(z)|}{(1+\|z\|)^{2}} \leq|f(z)| \leq \frac{|I(z)|}{(1-\|z\|)^{2}}
$$

The bounds (9) are sharp. 
Proof. For $z \in \gamma(I) \cap \Omega$ the inequality (9) holds by Corollary 1. So let us assume that $z \in \chi(I) \cap \Omega$ and $F_{w}$ is defined by (4). Using the growth theorem for the class $S^{c c}$ (see [16], [6]), we get

$$
\frac{|\zeta|}{(1+|\zeta|)^{2}} \leq\left|F_{w}(\zeta)\right| \leq \frac{|\zeta|}{(1-|\zeta|)^{2}}, \quad|\zeta|<1
$$

If we set $w=\frac{z}{\|z\|}$, then $w \in \chi(I) \cap \partial \Omega$ and by (4) we have

$$
\frac{\frac{1}{\|z\|}|\zeta||I(z)|}{(1+|\zeta|)^{2}} \leq\left|f\left(\zeta \frac{z}{\|z\|}\right)\right| \leq \frac{\frac{1}{\|z\|}|\zeta||I(z)|}{(1-|\zeta|)^{2}}
$$

so for $\zeta=\|z\|$ we obtain (9).

Now we shall prove the sharpness of bounds (9). Let $z$ be an arbitrarily fixed point from $\Omega$ and $w=\frac{z}{\|z\|}$. For $\varepsilon= \pm 1$ we consider the function

$$
f_{\varepsilon}(x)=\frac{I(x)}{\left(1-\varepsilon Z_{w}^{*}(x)\right)^{2}}, \quad x \in \Omega
$$

where $Z_{w}^{*} \in\left(\mathbf{C}^{n}\right)^{*}$ fulfils (5). Putting $\varepsilon=1$ we obtain

$$
\left|f_{1}(z)\right|=\frac{|I(z)|}{\left|1-Z_{w}^{*}(z)\right|^{2}}=\frac{|I(z)|}{\left|1-\|z\| Z_{\frac{z}{* z \|}}^{*}\left(\frac{z}{\|z\|}\right)\right|^{2}}=\frac{|I(z)|}{(1-\|z\|)^{2}}
$$

and the upper bound is achieved.

The proof that equality in the lower bound is attained for the function $f_{-1}$ is similar.

Theorem 3. If $f \in S^{c c}(\Omega), z \in \Omega$, then

$$
\frac{|I(z)|(1-\|z\|)}{(1+\|z\|)^{3}} \leq|D f(z)(z)| \leq \frac{|I(z)|(1+\|z\|)}{(1-\|z\|)^{3}} .
$$

The above estimate is sharp.

Proof. Let $z \in \gamma(I) \cap \Omega$. Then $I(z)=0$. Simultaneously $D f(z)(z)=0$, because $D f(z)(z)=I(z)+\sum_{m=2}^{\infty} m P_{m, f}(z)$ and by $(7) P_{m, f}(z)=0$. Thus we obtain equality in (11).

Let now $z \in \chi(I) \cap \Omega$ be arbitrarily fixed. Then for a function $F_{w}$ defined by (4) we get

$$
F_{w}^{\prime}(\zeta)=\frac{1}{I(w)}(D f(\zeta w)(w))
$$

From the distortion theorem for the class $S^{c c}$ (see [16], [6]) we have

$$
\frac{1-|\zeta|}{(1+|\zeta|)^{3}} \leq\left|F_{w}^{\prime}(\zeta)\right| \leq \frac{1+|\zeta|}{(1-|\zeta|)^{3}}, \quad|\zeta|<1
$$


Hence, for $w=\frac{z}{\|z\|}$ we obtain

$$
\frac{1-|\zeta|}{(1+|\zeta|)^{3}}\left|I\left(\frac{z}{\|z\|}\right)\right| \leq\left|D f\left(\zeta \frac{z}{\|z\|}\right)\left(\frac{z}{\|z\|}\right)\right| \leq \frac{1+|\zeta|}{(1-|\zeta|)^{3}}\left|I\left(\frac{z}{\|z\|}\right)\right| .
$$

Putting $\zeta=\|z\|$ we obtain (11).

To show the sharpness in (11) let us consider the function $f_{\varepsilon}, \quad \varepsilon= \pm 1$ defined in (10). For $\varepsilon=1$ we obtain

$$
D f_{1}(x)(x)=\frac{I(x)\left(1+Z_{w}^{*}(x)\right)}{\left(1-Z_{w}^{*}(x)\right)^{3}} .
$$

Putting $x=z$ we have

$$
D f_{1}(z)(z)=\frac{I(z)\left(1+\|z\| Z_{\|z\|}^{*}\left(\frac{z}{\|z\|}\right)\right)}{\left(1-\|z\| Z_{\| z}^{*}\left(\frac{z}{\|z\|}\right)\right)^{3}}=\frac{I(z)(1+\|z\|)}{(1-\|z\|)^{3}} .
$$

Analogously for $\varepsilon=-1$ we prove that for the function $f_{-1}$ we obtain equality in the lower bound.

The following theorem is a generalization of the above result.

Theorem 4. Let $n \in \mathbf{N}$ and $0 \neq z \in \Omega$. If $f \in S^{c c}(\Omega)$, then

$$
\frac{|I(z)|(n-\|z\|)}{(1+\|z\|)^{(n+2)}} \leq \frac{\left|D^{(n)} f(z)(z, \ldots, z)\right|}{n !\|z\|^{n-1}} \leq \frac{|I(z)|(n+\|z\|)}{(1-\|z\|)^{(n+2)}} .
$$

The above bounds are sharp and beeing attained by functions (10).

THEOREM 5. If $f \in S^{c c}(\Omega)$ is associated with $h \in S^{c}(\Omega)$ by (3), then

$$
\operatorname{Re} \frac{f(z)}{h(z)}>0, \quad z \in \chi(I) \cap \Omega .
$$

The proof is based on the following $n$-dimensional version of Jack Lemma (compare [4], [7]).

LEMMA 1. Let $f: \Omega \rightarrow \mathbf{C}$ be a holomorphic function such that $f(0)=0$ and

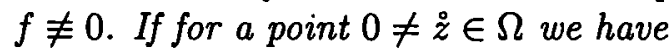

$$
|f(\dot{z})|=\max _{\|z\| \leq\|\tilde{z}\|}|f(z)|,
$$

then there exists $s \geq 1$ such that

$$
\frac{D f(\dot{z})(\stackrel{\circ}{z})}{f(\dot{z})}=s .
$$

Proof of the lemma. We will show that the function

$$
F(\zeta)=f\left(\zeta \frac{\dot{z}}{\|\dot{z}\|}\right), \quad \zeta \in \Delta
$$

satisfies the assumptions of classical Jack Lemma ([8]). Indeed, this function is holomorphic in $\Delta, F(0)=0$ and $F \not \equiv 0$. Moreover $\left|F\left(\zeta_{0}\right)\right|=$ 
$\max _{|\zeta| \leq\left|\zeta_{0}\right|}|F(\zeta)|$ for $\zeta_{0}=\|\dot{z}\|$. Indeed, from the assumptions of the lemma and the definition of $F$ we have

$$
\begin{aligned}
\left|F\left(\zeta_{0}\right)\right| & \leq \max _{|\zeta| \leq\left|\zeta_{0}\right|}|F(\zeta)|=\max _{|\zeta| \leq\left|\zeta_{0}\right|}\left|f\left(\zeta \frac{\dot{z}}{\|\dot{z}\|}\right)\right| \leq \\
& \leq \max _{\|z\| \leq\|\dot{z}\|}|f(z)|=|f(\dot{z})|=|F(\|\dot{z}\|)|=\left|F\left(\zeta_{0}\right)\right| .
\end{aligned}
$$

In view of Jack Lemma there exists $s \geq 1$ such that

$$
\zeta_{0} \frac{F^{\prime}\left(\zeta_{0}\right)}{F\left(\zeta_{0}\right)}=s
$$

Then, by the computation of the derivative of $F$ we obtain

$$
s=\frac{\zeta_{0} D f\left(\zeta_{0} \frac{\dot{z}}{\|\dot{z}\|}\right)\left(\frac{\dot{z}}{\|\dot{z}\|}\right)}{f\left(\zeta_{0} \frac{\dot{z}}{\|\dot{z}\|}\right)}
$$

This completes the proof, because $\zeta_{0}=\|\dot{z}\|$.

Proof of Theorem 5. It is sufficient to consider $f \in S^{c c}(\Omega)$ such that $f \neq h$. Since $h \in S^{c}(\Omega)$, we have $h(z) \neq 0$ and $D h(z)(z) \neq 0$ for $z \in \chi(I) \cap \Omega$ (see [10]). Denoting

$$
\frac{f(z)}{h(z)}=\frac{1+q(z)}{1-q(z)} \quad \text { for } \quad q(z) \neq 1, z \in \Omega,
$$

we can deduce that the function $q$ is holomorphic in $\Omega, q(0)=0$ and $q \not \equiv 0$. It is sufficient to prove that $|q(z)|<1$ for $z \in \chi(I) \cap \Omega$. In the oposite case, there exists $\dot{z} \in \chi(I) \cap \Omega$ such that

$$
1=|q(\stackrel{\gtrless}{)})|=\max _{\|z\| \leq\|\hat{z}\|}|q(z)| \text {. }
$$

In view of Lemma 1 , we conclude that there exists a real number $s$ such that

$$
\frac{D q(\dot{z})(\dot{z})}{q(\dot{z})}=s \geq 1
$$

From (12) we obtain

$$
D f(z)(z)=\frac{1+q(z)}{1-q(z)} D h(z)(z)+h(z) \frac{2 D q(z)(z)}{(1-q(z))^{2}}, \quad z \in \chi(I) \cap \Omega .
$$

Hence

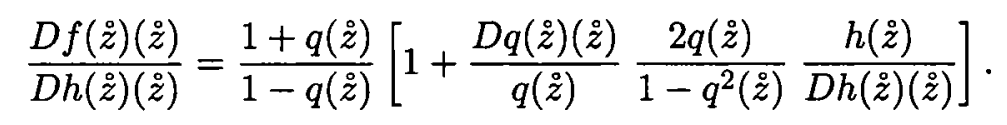

Since $|q(\dot{z})|=1$, so $\operatorname{Re} \frac{1+q(\dot{z})}{1-q(\bar{z})}=0$. Putting $\frac{1+q(\dot{z})}{1-q(\dot{z})}=i y$, where $y$ is a real 
number, we obtain $q(\stackrel{\circ}{z})=\frac{i y-1}{i y+1}$ and

$$
\frac{2 q(\dot{z})}{1-q^{2}(\dot{z})}=\frac{i\left(y^{2}+1\right)}{2 y}
$$

From the above considerations and in view of (13), we can rewrite (14) in the following form

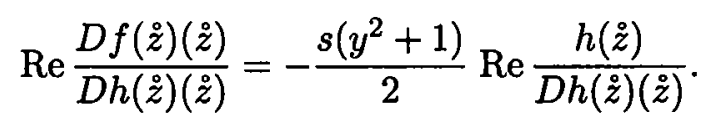

Since $h \in S^{c}(\Omega) \subset S^{*}(\Omega)$, we have $\operatorname{Re} \frac{D h(\dot{z})(\dot{z})}{h(\dot{z})}>0$. Therefore

$$
\operatorname{Re} \frac{D f(\stackrel{\circ}{z})(\stackrel{\circ}{z})}{D h(\check{z})(\stackrel{\circ}{z})}<0,
$$

which (by (3)) contradicts the fact that $f \in S^{c c}(\Omega)$.

Theorem 5 we can prove also using a modified version of the Sakaguchi Lemma (see [15]).

\section{References}

[1] I. I. Bawrin, Klassy golomorfnych funkcyj mnogich kompleksnych peremennych $i$ ekstremalnyje voprosy dla etich klassow funkcyj, "Nauka" Moskwa, (1976).

[2] M. Bier nacki, Sur la representation conforme des domaines lineairement accessibles, Prace Mat.-Fiz., 44 (1936), 293-314.

[3] K. Dobrowolska, I. Dziubiński, Of starlike and convex functions of many variables, Demonstratio Math. 11(2), (1978), 545-555.

[4] K. Dobrowolska, P. Liczberski, On some differential inequalities for holomorphic functions of many variables, Demonstratio Math. 14(2), (1981), 383-398.

[5] I. Dziubiński, R. Sitarski, On classes of holomorphic functions of many variables starlike and convex on some hipersurfaces, Demonstratio Math. 13(3), (1980), 619 632.

[6] A. W. Goodman, Univalent functions, Mariner Publishing Company 1983.

[7] S. Gong, S. S. Miller, Partial differential subordinations and inequalities defined on complete circular domains, Comm. Partial Differential Equations, 11 (1986), No. 11, 1243-1255.

[8] I. S. Jack, Function starlike and convex of order alpha, J. London Math. Soc. 3 (1971), 469-474.

[9] Z. J. Jakubowski, J. Kamiński, On some classes of Mocanu-Bazilevic functions, Acta Univ. Lodz., Folia Math., 5 (1992), 39-61.

[10] E. Janiec, On starlike and convex maps of a Banach space into the complex plane, Demonstratio Math. 27 (1994), 511-516.

[11] W. Kaplan, Close-to-convex schlicht functions, Michigan Math. J. 1 (1952) 169-185.

[12] Z. Lew andowski, Sur l'identite de certaines classes de fonctions univalentes, part I, Ann. Univ, M. Curie-Skłodowska, 12, (1958), 131-146; part II, ibid. 14 (1960), $19-46$. 
[13] P. Liczberski, On Bavrin families of holomorphic maps of Banach spaces into the complex plane, Zesz. Nauk. Polit. Rzesz, 48 (1988), 77-85.

[14] W. Rudin, Function Theory in the Unit Ball, Springer-Verlag, New York Inc., 1980.

[15] K. Sakaguchi, On certain univalent mapping, J. Math. Soc. Japan 11 (1959), 72-75.

[16] G. Schober, Univalent Functions-Selected Topics, Springer-Verlag, New York Inc., 1975.

FACULTY OF COMPUTER SCIENCE

ACADEMY OF HUMANITIES AND ECONOMICS IN LÓDŹ

Rewolucji 1905r. 64

90-222 LODŹ, POLAND

E-mail: amarchlewska@wshe.lodz.pl

Received February 25, 2004; revised version April 5, 2005. 